\title{
Operational Amplifiers Revisited for Low Field Magnetic Resonance Relaxation Time Measurement Electronics ${ }^{\dagger}$
}

\author{
Najlaa K. Almazrouei, Michael I. Newton * and Robert H. Morris \\ School of Science and Technology, Nottingham Trent University, Clifton Lane, Nottingham NG11 8NS, UK; \\ najlaa.almazrouei022016@my.ntu.ac.uk (N.K.A.); rob.morris@ntu.ac.uk (R.H.M.) \\ * Correspondence: Michael.newton@ntu.ac.uk; Tel.: +44-115-848-3365 \\ + Presented at the 6th International Electronic Conference on Sensors and Applications, 15-30 November \\ 2019; Available online: https://ecsa-6.sciforum.net/.
}

Published: 14 November 2019

\begin{abstract}
Advances in permanent magnet technology has seen more reports of sensor applications of low field magnetic resonance. Whilst most are either in the 10-20 MHz range or in the earth's field, measurements at below $1 \mathrm{MHz}$ are beginning to become more widespread. This range is below the need for careful radio frequency electronics design but above the audio domain and represents an interesting cross over. Many commercial spectrometers do not include the pulse power amplifier, duplexer and preamplifier as these depend on the frequency range used. In this work we demonstrate that, with the current specifications of the humble operational amplifier, the most simple form of an inverting design using only two resistors and decoupling, can effectively provide this 'front end' electronics. The low powers used mean crossed Ge diodes provide an excellent duplexer and it is suitable for battery powered applications.
\end{abstract}

Keywords: operational amplifier; opamp; NMR; relaxation time; electronics

\section{Introduction}

Nuclear magnetic resonance (NMR) finds many applications from the chemical finger prints of NMR spectroscopy [1] to the whole body magnetic resonance imaging (MRI) scanners found in many hospitals [2]. Two of the parameters that can be used in the production of MRI images are the spinlattice $\left(T_{1}\right)$ and spin-spin $\left(T_{2}\right)$ relaxation times and measurements of these have been used in a wide variety of sensor applications that may use permanent magnets and less expensive electronics [3-5]. The electronics, commonly referred to as the console, for pulsed NMR systems are designed to be wide band and highly programmable spectrometers with only the power amplifier, duplexer and preamplifier specific to a narrow frequency range [6]. Off the shelf electronics for the power amplifier and preamplifier are often designed to be capable of handling much wider frequency ranges than are required for a specific application and for high power than required, which in turn requires more careful and expensive design of the duplexer. A typical system of off the shelf components may consist of a ZHL-3A+ power amplifier from Mini-Circuits (US\$229) fed to the input to an nmrservice.de transcoupler (duplexer) that operates from 0.7 to $1 \mathrm{MHz}$ (US\$735) and the return signal amplified using a Mini-Circuits ZFL-500LN (US\$93) giving a cost of more than US\$1000. Advances in permanent magnet technology has seen report of applications of low field unilateral magnets below $20 \mathrm{mT}$ corresponding to frequencies below $1 \mathrm{MHz}$ [7]. In this work we demonstrate that the current generation of operational amplifiers, in the form of a simple inverting design, can effectively 
provide the power and preamplifiers in this frequency range and at a fraction of the cost of commercial components.

\section{Materials and Methods}

The schematic diagram of the power amplifier, duplexer and preamplifier circuits are shown in Figure 1. The signal produced by the Spincore RadioProcessor board [8] is fed via a decoupling capacitor to a simple inverting opamp circuit using a Linear Technology LT1363 [9]. This opamp has a $70 \mathrm{MHz}$ gain bandwidth product and can operate from a $+15 \mathrm{~V}$ to $-15 \mathrm{~V}$ supply. To produce the supply voltages of both amplifiers we used a pair of TRACO POWER TSRN 1-24150 dc to dc converter/switching regulators. This allowed a single dc supply of between $16.5 \mathrm{~V}$ and $42 \mathrm{~V}$ to be used, making it suitable for battery power applications. The resistors used in the amplifier gave a gain of around 12 and a bandwidth of $5.7 \mathrm{MHz}$. The output of the amplifier was decoupled and fed to a pair of back to back Ge diodes, forming part of the duplexer, which effectively disconnects the transmitter output from the tuned circuit and preamplifier during the receive part of the sequence to prevent leakage signal from the rf output of the SpinCore entering the detection circuit [6]. The sample was placed in a coil which formed part of a tuned circuit with a tuning and matching capacitor and set to give a resonant frequency of $0.808 \mathrm{MHz}$. The coil was placed in between the poles of an electromagnet that could have the field changed by means of a dc constant current power supply; the correct field for the resonant frequency used was generated by a current of $0.335 \mathrm{~A}$.

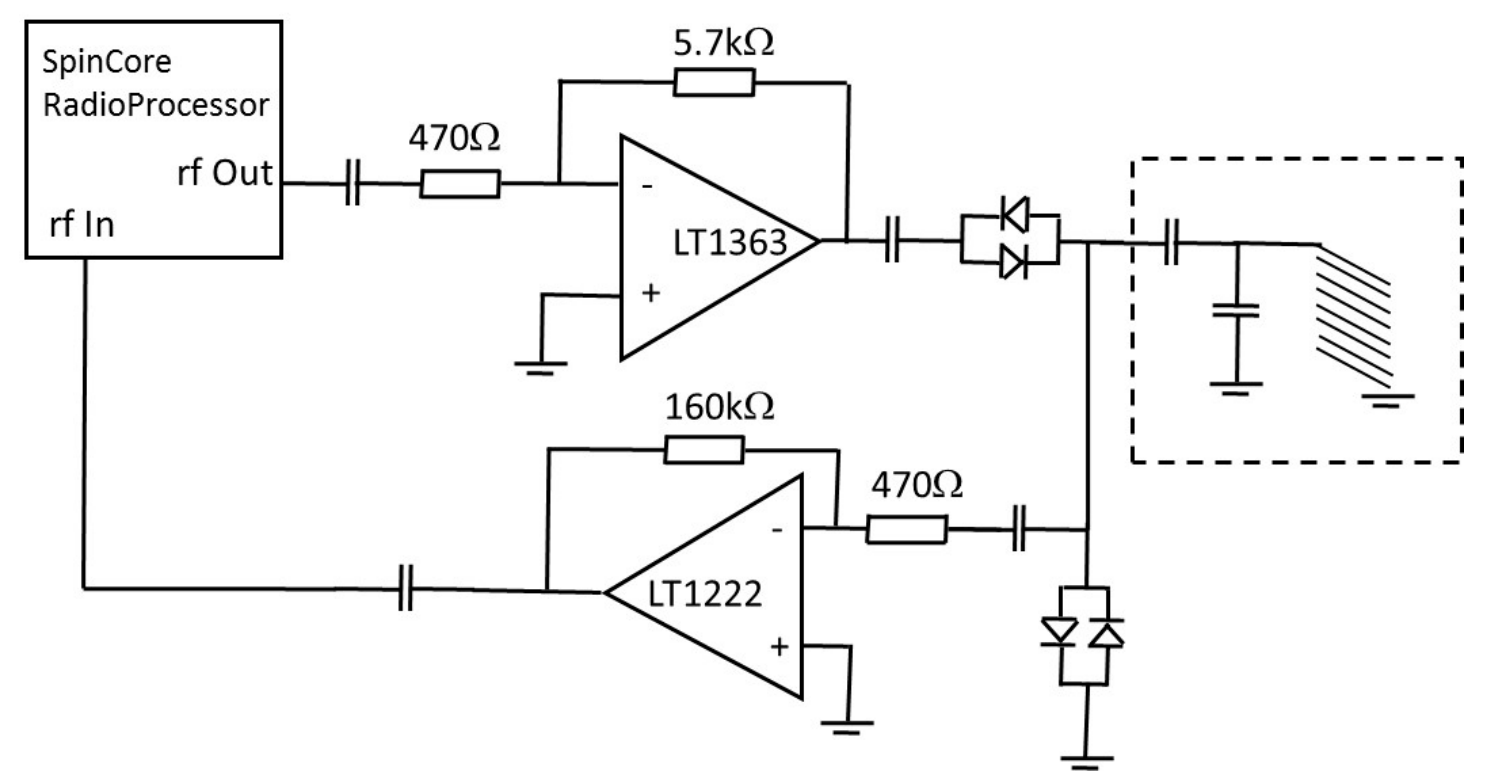

Figure 1. Schematic diagram of the power amplifier, duplexer and preamplifier circuits. All capacitors are $0.1 \mu \mathrm{F}$ and the diodes are Ge. The dotted line marks the sample coil with the tuning and matching circuit. Both amplifiers are powered with $+15 \mathrm{~V}$ and $-15 \mathrm{~V}$ from dc-dc converters.

The NMR signal returning from the coil is orders of magnitude smaller than the signal from the LT1363. To protect the preamp a pair of back to back Ge diodes were connected to ground so that the maximum input to the preamp was limited to $0.2 \mathrm{~V}$; note that this is more effective protection than the $0.6 \mathrm{~V}$ offered by $\mathrm{Si}$ diodes. This was fed via a decoupling capacitor to a simple inverting opamp circuit using a Linear Technology LT1222 [10]. This has a $500 \mathrm{MHz}$ gain bandwidth product and again can operate from a $+15 \mathrm{~V}$ to $-15 \mathrm{~V}$ supply however it has a smaller current sourcing capability than the LT1363. The resistors used in the amplifier gave a gain of around 340 and a bandwidth of 1.47 $\mathrm{MHz}$ which effectively acts like a low pass filter for reducing high frequency noise returning to the Spincore. The circuit was tested with ThorLabs EF508 $1 \mathrm{MHz}$ low pass filters on the input and output of the LT1222, these were found to make little difference.

The height and diameter of the coil were both $20 \mathrm{~mm}$ and made up of 3 layers of 30 turns of Litz wire. The Litz wire had 81 strands of $0.04 \mathrm{~mm}$ diameter copper wrapped in silk giving an overall wire 
diameter of $0.67 \mathrm{~mm}$. A sample tube of $14 \mathrm{~mm}$ diameter was filled with olive oil as the test liquid used for all the measurements. For all measurement a CPMG sequence was used with the amplitude of the 180 pulses being twice the amplitude of the 90 pulse but of the same duration.

\section{Results}

Figure 2 shows an echo train of 32 spin echoes with TE of $3500 \mu$ s, TR of $312 \mathrm{~ms}$, operating at $0.808 \mathrm{MHz}$ with a CPMG sequence [11] and with 1024 averages. A fit to the echo peaks will give a value for $\mathrm{T}_{2}$ (effective) and a usable value for this could be achieved using an order of magnitude less averages than presented. Note that, as is often the case for low frequency pulsed NMR the initial Hahn echo is slightly smaller than the subsequent echo.

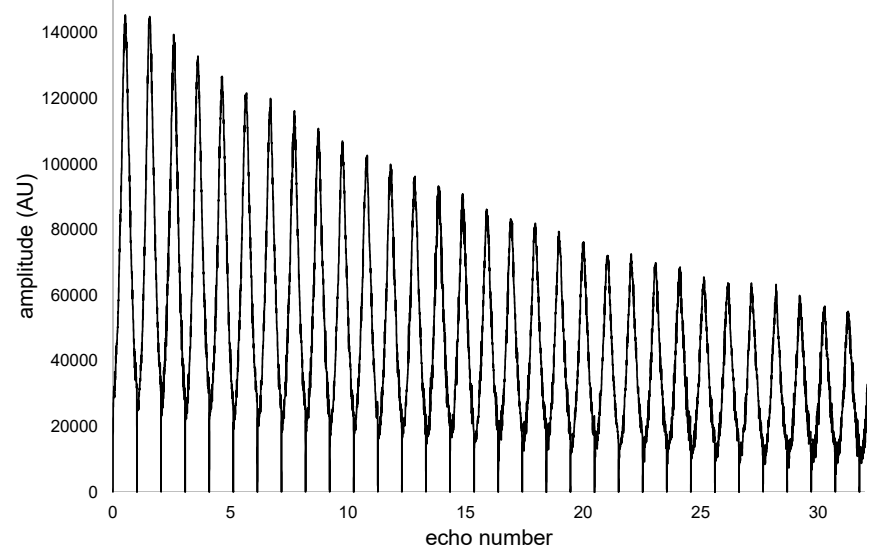

Figure 2. A series of spin echoes measured using the opamp electronics. A fit to the peaks provides an estimate for the value of $\mathrm{T}_{2}$ (effective).

Figure 3 shows a find 90 optimization plot where the signal integral is measured over a range of time values for the pulse length. In this case the TE was $1500 \mu \mathrm{s}$, TR $248 \mathrm{~ms}$ and 128 averages for the 32 echoes. The peak represents the optimum value to be used for the pulse length and in this case is $40 \mu$ s. Initially we tried an LT1222 as the power amplifier from a $+12 \mathrm{~V}$ and $-12 \mathrm{~V}$ power supply and for this the optimum 90 was found to be $45 \mu$ indicating that as expected when the power is increased, the required 90 is reduced. Using the optimum length for the 90 we can then provide an estimate to T1 by running an integral, like the find 90, but with changing repetition time TR and fitting an exponential to the data. In Figure 4 we show this with TR from 25 ms to 700 ms, again with 32 echoes and 128 averages per point.

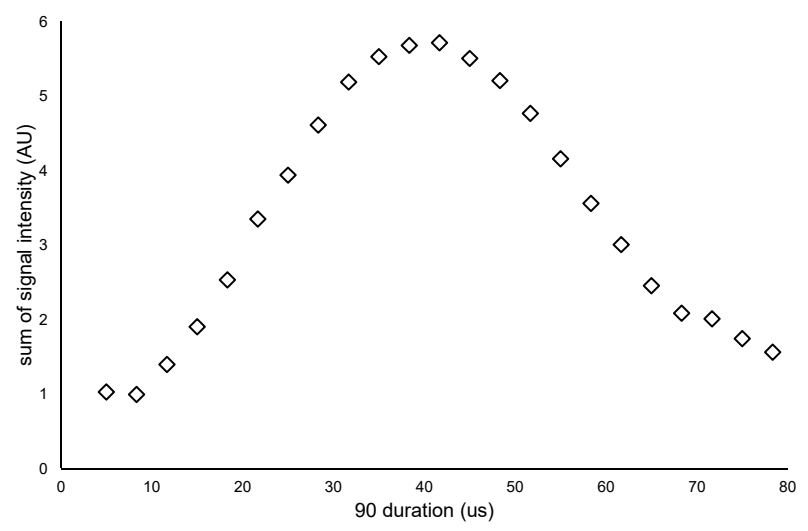

Figure 3. A calibration of the duration of the 90 pulse length showing the optimum value at $40 \mu \mathrm{s}$. 


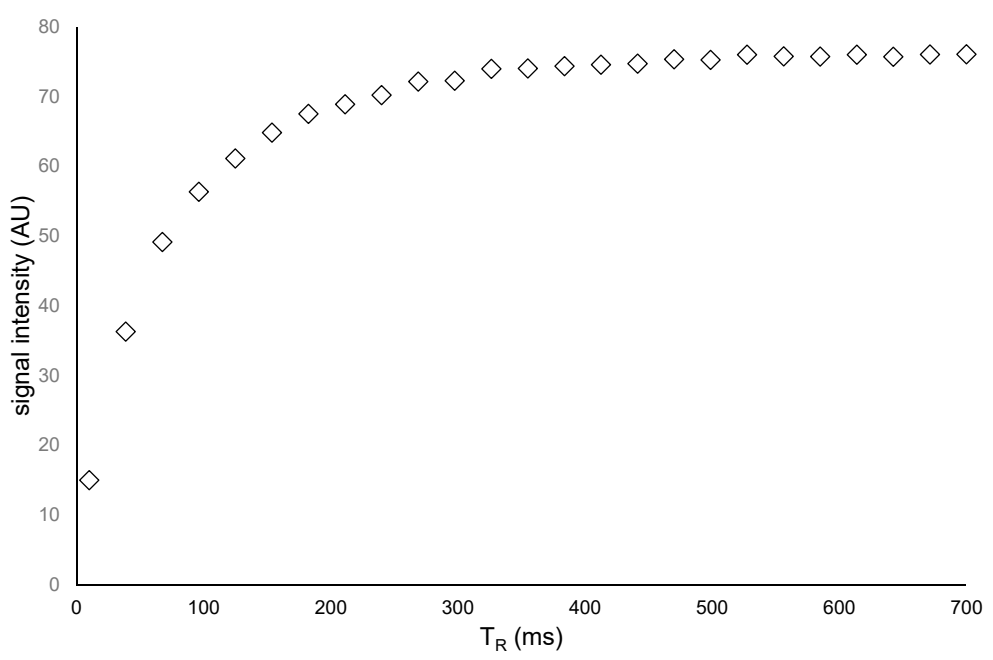

Figure 4. The signal intensity as a function of repetition time. A fit to the data can be used to give an estimate for the spin lattice relaxation time $\mathrm{T}_{1}$.

\section{Discussion}

In the frequency range just below $1 \mathrm{MHz}$ we have demonstrated that we can still get excellent results with much lower power, compared to commercial amplifiers, with the use of simple opamp circuits making it very applicable to battery powered applications. The data we have presented used a reasonably homogeneous magnetic field from a laboratory electromagnet. For fully battery operated applications this would need to use permanent magnets which may give less homogeneity and may then require greater power amplification that can currently be provided by the LT1363. The component cost of both amplifier circuits total less than US\$20 compared to more than US\$1000 for off the shelf radio frequency specified commercial components that also cover this frequency range. In addition, there is no requirement for additional low pass filtering as the bandwidth of the amplifier circuit itself excludes passing radio frequencies.

Author Contributions: Conceptualization and Methodology, M.I.N. and R.H.M.; Software, R.H.M.; Investigation, M.I.N. and N.K.A.; Writing-Original Draft Preparation, M.I.N.; Writing-Review \& Editing, R.H.M. and N.K.A.

Funding: N.K.A. gratefully acknowledges the Ministry of Higher Education and Scientific Research (MOHESR) in the United Arab Emirates for funding and the Dubai Health Authority (DHA) for study leave.

Conflicts of Interest: The authors declare no conflict of interest.

\section{References}

1. Weber, U.; Thiele, H. NMR Spectroscopy: Modern Spectral Analysis, 1st ed.; Wiley VCH: Weinheim, Germany, 1998.

2. Dale, B.M.; Brown, M.A.; Semelka, R.C. MRI: Basic Principles and Applications; Wiley-Blackwell: Hoboken, NJ, USA, 2015.

3. Blumich, B.; Perlo, J.; Casanova, F. Mobile single-sided NMR. Prog. Nucl. Magn. Reson. Spectrosc. 2008, 52, 197-269, doi:10.1016/j.pnmrs.2007.10.002.

4. Blümich, B. Introduction to compact NMR: A review of methods. Trends Anal. Chem. 2016, 83, 2-11, doi:10.1016/j.trac.2015.12.012.

5. Kirtil, E.; Cikrikci, S.; McCarthy, M.J.; Oztop, M.H. Recent advances in time domain NMR \& MRI sensors and their food applications. Curr. Opin. Food Sci. 2017, 17, 9-15, doi:10.1016/j.cofs.2017.07.005.

6. Fukushima, E.; Roeder, S.B.W. Experimental Pulse NMR: A Nuts and Bolts Approach, 1st ed.; Westview Press: Boulder, CO, USA, 1981.

7. Dabaghyana, M.; Muradyan, I.; Hrovat, A.; Butler, J.; Fredrick, E.; Zhou, F.; Kyriaziz, A.; Hardin, C.; Patz, S.; Hrovat, M. A portable single-sided magnet system for remote NMR measurements of pulmonary function. NMR Biomed. 2014, 27, 1479-1489, doi:10.1002/nbm.3149. 
8. SpinCore RadioProcessor Board. Available online: http://www.spincore.com/products/RadioProcessor/ (accessed on 27 February 2019).

9. LT1363 Data Sheet. Available online: https://www.analog.com/media/en/technical-documentation/datasheets/1363fa.pdf (accessed on 28 February 2019).

10. LT1222 Data Sheet. Available online: https://www.analog.com/media/en/technical-documentation/datasheets/1222fc.pdf (accessed on 28 February 2019).

11. Meiboom, S.; Gill, D. Modified Spin-Echo Method for Measuring Nuclear Relaxation Times. Rev. Sci. Instrum. 1958, 29, 688, doi:10.1063/1.1716296.

(C) 2019 by the authors. Licensee MDPI, Basel, Switzerland. This article is an open access article distributed under the terms and conditions of the Creative Commons Attribution (CC BY) license (http://creativecommons.org/licenses/by/4.0/). 